\title{
LA HILERA DEL VALOR SOCIAL Y EL PAPEL DEL ESTADO
}

\section{Clara Caselli}

Paradigma del valor e hilera del valor

T I punto de partida de este artículo es la idea de la producción del valor. La dignidad de cualquier iniciativa que tenga una naturaleza económica y lo que legitima su existencia es la capacidad de producir valor en el ambiente económico y social, todos los operadores buscan un valor en el desarrollo de su actividad y existe un sistema del valor porque el empresario produce valor para sí mismo y para todos los que entran en relación con su empresa. De esta manera, se forma una cadena o una hilera del valor en la economía y en la sociedad.

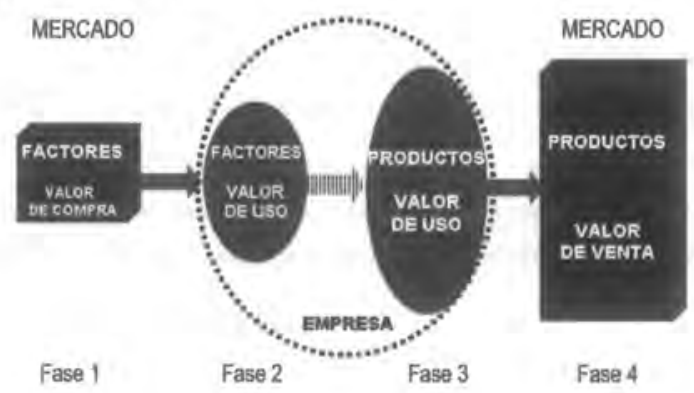


También los actores que no son empresas tienen su manera de producir valor y lo producen desarrollando relaciones entre ellos y con las empresas. El paradigma del valor en todas sus fases es un paradigma social. En las fases 1 y 4 el sistema individual de creación del valor se relaciona con el mercado; con los proveedores, por un lado, y con los clientes, por otro; en ambos casos, todo acontece en el marco de la presencia de la empresa en la sociedad hacia la cual se dirige la propuesta social que la empresa misma elabora. En las fases 2 y 3 , la dinámica social se desarrolla básicamente al interior de la empresa $y$ se trata de una dinámica que implica personas, conocimientos, informaciones, relaciones organizacionales formales e informales.

La dinámica de producción del valor — como hemos vistosiempre se caracteriza por dos aspectos: uno que riene un precio, un valor de mercado, y otro que no lo tiene y es el motor de todos los procesos de innovación y de desarrollo.

Tratemos ahora de describir el proceso de producción del valor en la sociedad, para entender también como ha cambiado el papel del Estado y cuál debería ser su función en la sociedad contemporánea.

\section{Fase 1: el mercado centro de la vida económica}

Esta fase, que históricamente llega hasta los años 20, se caracteriza por un proceso de creación del valor concentrado en el mercado y en las empresas, El mercado es la "mano invisible» que arregla todo. Si cada uno de los actores tiene como fin la maximización de su propio interés, el resultado final es la maximización de la urilidad para toda la sociedad. En otras palabras, el bien común es la suma de las funciones de utilidad individuales; una suma de *egoísmos" produce el bien general.

El papel del Estado en este modelo es muy marginal; define reglas mínimas del juego, garantiza la defensa y el orden público. 


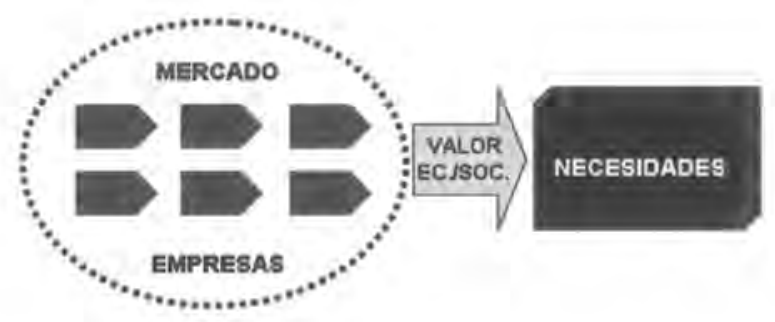

Pero el mercado fracasa por varios motivos:

- Existen bienes de naturaleza pública. El mercado no logra producir todos los bienes públicos que la sociedad necesita, es decir, los bienes para los cuales no hay competencia ni rivalidad entre los individuos porque el uso por parte de uno no excluye el uso por parte de otros. Por ejemplo, este es el caso de las carreteras, de la defensa, de la justicia. No existe un mercado de esos bienes, porque nadie puede ser excluido.

- Existen bienes sin precio. A veces la actividad económica de una empresa produce consecuencias sobre otras empresas o personas, pero estos efectos no pueden ser medidos, porque no hay precios que puedan medirlos. Este es el caso de la contaminación del medio ambiente: existe un perjuicio económico, pero no hay un precio. En estos casos, el mercado - por su naturaleza - no tiene la capacidad de preocuparse por las consecuencias de sus acciones (mejor, de las acciones individuales de las empresas), porque esto implicaría un costo adicional del cual no se percibe una inmediata rentabilidad y, por lo tanto, no hay quien pueda hacerse cargo.

- A veces el mercado no funciona porque las informaciones relativas a la calidad, a la cantidad y al precio de los bienes no circulan bien: unos sujetos las poseen y a otros les faltan. Si en 
un mercado hay alguien que sabe mucho y otros que no saben nada, las relaciones económicas no están equilibradas y no son eficientes (por ejemplo, este es el caso del mercado laboral, del mercado del crédito, del mercado de los seguros).

- Los mercados tienen muchas imperfecciones: no siempre hay una verdadera competencia entre las empresas, al contrario, prevalecen empresas monopolistas que pueden aprovecharse de su posición para ganar más; no siempre el acceso al mercado es posible para todos y se encuentran obstáculos a la entrada, etc.

Todos estos casos nos hacen entender que no siempre la "mano invisible» equilibra mágicamente las cosas. Pero no podría ser diferente. ¿Cómo es posible que la suma de los "egoísmos" individuales produzca un bienestar general?

En conclusión, la mano invisible del mercado logra producir valor económico, pero fracasa cuando se trata de producir valor social y especialmente de construir la justicia y la equidad en la sociedad.

\section{Fase 2: el Estado como remedio a los fracasos del mercado}

Para solucionar los problemas creados por el mercado, se pensó en un fuerte crecimiento del papel del Estado. A partir del período entre las dos guerras mundiales, el Estado interviene masivamente en la escena económica por medio de la creación de empresas públicas y financiaciones a las empresas privadas y desarrolla también una actividad social por medio de una actividad directa en los sectores de la educación, salud, trabajo, cultura, etc.

Nace la idea del "estado de bienestar" (welfare state) que extiende su influencia a todos los sectores de la vida económica y social, desempeñando actividades de las cuales antes se ocupaba el 
mercado. Lo que ocurre es que en esta extensión de actividad, no siempre la acción pública es más eficiente que la de las empresas y, además, la fuerte y masiva presencia social del Estado toma los rasgos de un control social y político de la población (se brindan servicios no según un criterio de justicia y eficiencia social, sino simplemente porque el beneficiario es un elector potencial). Se manifiesta evidente la pretensión del poder que quiere dominar la vida de la gente y determinar los valores y la cultura. El estado de bienestar en sus realizaciones más avanzadas es el único productor de valor social que reparte como si fuera una lluvia a todo el universo según una relación entre la institución y el individuo que va de arriba hacia abajo y tiene un carácter anónimo porque no es una relación entre persona y persona. En este contexto, mientras el Estado domina la vida pública, las relaciones interpersonales se desarrollan según la lógica del instinto y del interés particular.

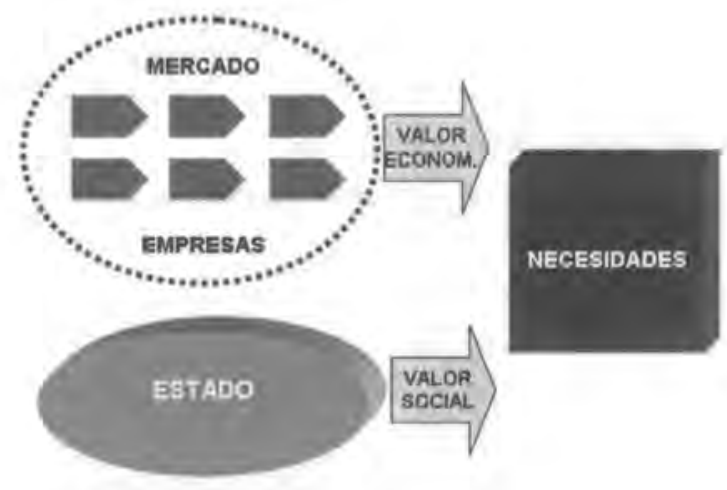

En síntesis, es muy evidente que - si es verdad que el mercado no funciona - también el Estado fracasa y no logra producir más justicia de la que produce el mercado. Los motivos son muchos: 
- No siempre los políticos y los funcionarios públicos toman decisiones y tienen comportamientos correctos, al contrario, a veces se aprovechan de su poder para conseguir más poder $\mathrm{y}$ también ganancias ilegales. Estos comportamientos son más probables si el papel del Estado es muy amplio, como enseña la experiencia de algunos países avanzados (Japón, Italia, etc.), o si el país no es muy desarrollado y la presión de las necesidades muy fuerte.

- Si es verdad que no siempre las decisiones tomadas gracias al mecanismo del mercado, es decir, mediante los precios, son racionales, no se entiende el motivo por el cual las decisiones políticas que se toman sin mecanismos que tengan una sólida base objetiva, deberían ser más racionales y eficientes.

- El sector público es mucho más burocratizado que el sector privado, porque no se mueve buscando una ganancia y no está sujeto a la competencia de las empresas. La presencia de la burocracia no elimina la asimetría informativa, al contrario, hay funcionarios que saben mucho y ciudadanos que están en una posición de inferioridad muy grande: se originan de esta manera nuevas formas de asimetría.

- Si el Estado dilata su actividad necesita más recursos y, por lo tanto, pide más impuestos, sustrayendo recursos a las empresas privadas y a los particulares que podrian usarlos de una manera diferente, quizás más eficiente.

- En la lógica del mercado, el objetivo es económico (el provecho propio). En la lógica del Estado, el objetivo es político, es decir, el crecimiento del poder, lo que implica una lógica que no es económica. Resulta probable que ascenso político y parsimonia no estén de acuerdo.

- No siempre las decisiones tomadas según el principio de la mayoria son verdaderamente democráticas. Hay una 
paradoja muy famosa, conocida como la paradoja de la mayoría. Supongamos que se tenga que tomar una decisión muy sencilla, como por ejemplo, pintar el muro de una casa: tenemos que escoger entre blanco, azul y rojo, además, queremos respetar el principio de la democracia y, por lo tanto, preguntamos a las tres familias que vivirán allí qué color prefieren. La familia número 1 prefiere el blanco al azul $\mathrm{y}$ al rojo, mientras que a la familia número 2 le agrada más el azul que el rojo y el blanco y la tercera familia indica en el orden rojo, blanco y azul. Bueno, entre el blanco y el azul, la mayoría prefiere el blanco; entre el azul y el rojo, el favorito es el azul. Por lo tanto, se podría concluir que el orden de preferencia por la mayoría es el siguiente: blanco, azul y rojo. Al contrario, dos familias prefieren el rojo al blanco.

- El desarrollo del Estado social, como ya se ha dicho, implica el uso de recursos económicos para brindar servicios que muchas veces no tienen una calidad alta y que, además, cuestan mucho. La experiencia de los países más desarrollados enseña con mucha evidencia las contradicciones de este modelo de Estado.

\section{Fase 3: la sociedad civil al centro de la hilera del valor}

Frente al fracaso del Estado, la pregunta interesante es si pueda existir un modelo diferente. En realidad, no hay una tercera via, sino simplemente una manera diferente de mirar a los actores y de imaginar su papel.

Antes que nada surge una pregunta ¿̇no se pueden imaginar más actores? Cuando un equipo de fútbol está perdiendo, el entrenador hace ingresar al campo de juego nuevos jugadores.

¿Hay algo entre el Estado y el mercado? La respuesta es afirmativa, ya que entre estos dos componentes, la sociedad civil juega 
un papel de gran preponderancia mediante todas sus modalidades de agregación y de intervención. Además de buscar a nuevos jugadores, se puede también cambiar el esquema de juego (y también el entrenador).

Por un lado, hay que pensar en las alianzas estratégicas entre las empresas que reducen el riesgo y la incertidumbre de los mecanismos de mercados y se dan reglas de conducta compartidas. A veces estas alianzas pueden generar mercados imperfectos que perjudican los consumidores, pero, en muchos casos, se trata de acuerdos que permiten a todos ser beneficiados (por ejemplo, redes de pequeñas empresas que permiten la salida directa a los mercados internacionales). Por otro lado, hay todas las iniciativas que nacen del deseo de hacer algo útil para los demás (por ejemplo, cooperativas, postas médicas, talleres, comedores, colegios, universidades, etc.), son iniciativas que nacen de valores e ideales y saben darse reglas y constituyen un verdadero tejido que posee un gran valor y un tremendo potencial de desarrollo. Muchas de estas iniciativas se desarrollan a partir de la iglesia, de los sacerdotes y de los laicos.

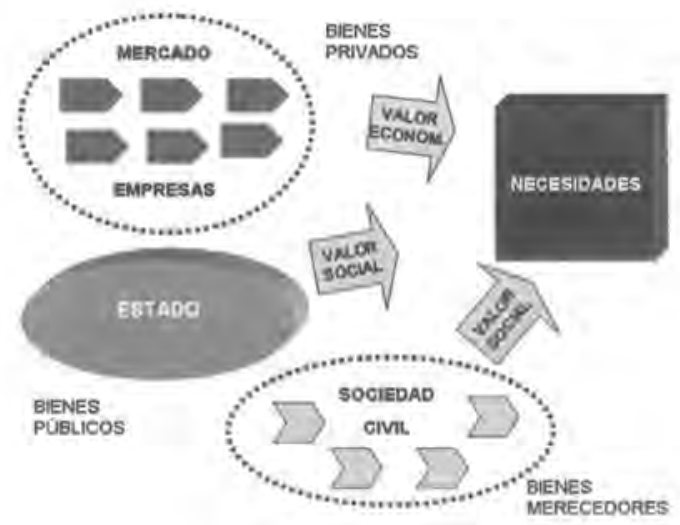


Como conclusión merece subrayar que la sociedad civil no es simplemente un remedio a los fracasos del mercado y del Estado; se trata de un modelo de sociedad rico y articulado basado en el protagonismo social.

El punto más interesante son las relaciones nuevas que pueden generarse entre la sociedad civil y el mercado, así como entre la sociedad civil y el Estado. Entre empresas privadas y sociedad se establece una continuidad y una colaboración, como es evidente en el caso de las empresas pequeñas y medianas que constituyen, en colaboración con la sociedad civil y los gobiernos locales, un tejido económico y social que puede ser una formidable ventaja competitiva y un modelo de desarrollo viable $y$ eficiente que respeta y valora todas las culturas y las tradiciones locales.

Resulta interesante también la colaboración entre el sector público y la sociedad civil: el Estado descubre una manera diferente de desempeñar sus funciones, es decir, un fin público se puede conseguir más eficiente y eficazmente de una manera indirecta, por medio de las formas solidarias que existen en la sociedad. El resultado va a ser un servicio que cuesta menos y, al mismo tiempo, satisface mejor las necesidades de la población, porque los mismos ciudadanos, que tienen la necesidad ( $y$, por lo tanto, la conocen bien), han colaborado en la construcción de la respuesta. En este tipo de modelo, no es necesario eliminar al mercado y al Estado, al contrario, ambos deben desarrollar un papel muy importante.

El mercado actúa como mecanismo de alocación eficiente de los recursos. Ya no es una mano invisible y casi milagrosa, sino el resultado de una construcción social. En este concexto, el estado tiene que desempeñar cuatro funciones muy importantes: 
1. Definir las ureglas del juegow y garantizar el respeto de las mismas.

2. Encargarse de las obras infraestructurales (físicas e informáticas) para adecuar el país a los estándares universales.

3. Brindar servicios para facilitar, apoyar y soportar la creatividad de la sociedad civil (pequeñas empresas, empresas no lucrativas, voluntariado, etc.).

4. Satisfacer usubsidiariamentew las necesidades de las comunidades con mayores carencias.

A raíz del comportamiento de todos los actores, se origina un sistema de producción del valor, que entra en dialéctica a escala internacional con los demás sistemas. Este tipo de dialéctica es muy importante para decidir qué tipo de globalización va a prevalecer en el mundo contemporáneo entre las dos alternativas de una globalización con homologación de todos los actores a de una que respeta y valoriza todas las identidades, las culturas y las tradiciones locales. 
LA HILERA DEL. VALOR SOCIAL Y EL PAPEL DEL. ESTADO

\section{Bibliografía}

Ambrosini, A. "L'economia morale può essere efficiente?». Impresa $e$ Stato, vol. 57, n. ${ }^{\circ} 8,1997$.

Anheier, H. K, "Il terzo settore in Europa: quattro tesi». Non Profit, n. $3,1999$.

Andreaus, M. «Un modello di osservazione economico-aziendale per le aziende non-profits. En Borzaga, C.; G. Fiorentini ; A. Matacena. Non-profit e sistemi di welfare. Roma: NIS, 1996.

Ascolr, U. (ed.), Il welfare futuro. Roma: Carocci, 1999.

Borzaga, C.; G. Fiorentini; A. Matacena. Non-profit e sistemi di welfare. Roma: NIS, 1996; Atti del Convegno AIDEA "Le aziende non profit tra stato e mercaton, Roma, 28/30 settembre 1995.

Borzaga, C; A. SAntuari. The Innovative Trends in the Non-profit Sector in Europe: the Emergence of Social Entrepreneurship, Conference Paper OECD/LEED Forum on Social Innovations, 2000.

Bowen, W. "Business leader senza fini di lucro", Harvard Business Review, maggio-giugno, 1995.

Caselli, Clara "Il non profit: aspetti aziendali". Persone of Imprese, n. ${ }^{\circ}$ 2, 1998.

— "Non profit: una totale dignitá di azienda». Persone Imprese \& Istituzioni, $\mathrm{n}^{\circ}{ }^{\circ} 1,2000$,

—Oltre la finanza etica». Persone Imprese \& Istituzioni, n.o 3, 2000. 
«Empresas y empresarios: camino al éxito y producción del valor económico y social". Studium Veritatis, año 3, n. ${ }^{\circ} 4-5,2002$.

Cavenago, D. Dirigere e governare una organizzazione non profit, Padova: Cedam, 1996.

Colombo, A.; S. Zaninelli. "Stato e formazioni sociali nell'Italia contemporanea: storia di una competenza negatam. En VitTAdiNi G., Sussidiarietá. La riforma possibile. Milano: Etaslibri, 1998.

Colozzı I.Y y A. Basst. Una solidarietà efficiente. Roma: NIS, 1995.

Cova, A. "La situazione italiana: una storia di non profit . En VITTADINI G. Il non profit dimezzato. Milano: Etaslibri, 1997.

FrorentinI, G. "Il contributo della teoria economica ai processi di privatizzazione dei servizi sociali. En BorzagA C.; FiorentinI G.; Matacena A. Non-profit e sistemi di welfare. Roma: NIS, 1996.

Merlo, A. "Gestire l'organizzazione no profit». En Pettinato S. (ed.). Gestire il no profit. Guida pratica alla gestione degli enti senza fine di lucro. Milano: Il Sole 24 Ore Librio, 1997.

Mirabil. M. L.; G. B. Sgritta. «Trasformazioni socio-demografiche e cambiamenti strutturali: le dimensioni della solidarietán. En Ascous U. Il welfare futuro. Roma: Carocci, 1999.

Pasquinelti, S. "Il Terzo settore nei diversi Welfare States europei». En Ascois U. Il welfare futuro. Roma: Carocci, 1999. 
Pearce, J.L. Volontariato. Milano: Cortina editore, 1994.

Petrinato S. Gestire il no profit. Guida pratica alla gestione degli enti senza fine di lucro. Milano: Il Sole 24 Ore Libri, 1997.

Salamon, L. M.; H. K. ANHEIER Il settore nonprofit in un confronto internazionale: profili e tipi. Universitá Cattolica del sacro Cuore, Centro di ricerche sulla cooperazione, Quaderni occasionali, n. ${ }^{\circ} 3$, 1994.

-Il setore non profit in una prospettiva comparata. Una panoramica. Universitá Cattolica del sacro Cuore, Istituto per la ricerca sociale, Quaderni occasionali, n. ${ }^{\circ}$ 6r 1994.

Global Civil society. Baltimore: Dimensions of the non profit sector, Johns Hopkins, 1999.

Santuari A. «Uno sguardo di insieme sulle esperienze straniere». En VitTAdini G. Il non profit dimezzato. Milano: Etas Libri, 1997.

SENN, L. «Autonomia, decentramento e sussidiarietá: una prospettiva economicay. Persone, Imprese \& Istituzioni, n. ${ }^{\circ}$ 1, 2000.

Sparrow, V. R. Melandru "Dal caso americano al caso italiano". En Vittadini G. Il non profit dimezzato. Milano: Etaslibri, 1997.

Tessitore, A. "Il profilo aziendale delle Organizzazioni Non Profits. Rivista italiana di Ragioneria e Economia Aziendale, n. ${ }^{\circ}$ 1-2, 1997.

Vittadini G. Il non profit dimezzato. Milano: Etas Libri, 1997. 
Vittadini G; M. Barea, La economia del non profit. Libre espresión de la sociedad civil. Madrid: Encuentro, 1999.

Zamagni, S. "La sussidiarietà, l'economia e la nuova carta costituzionale», En VitTadini G., Sussidiarietì, La riforma possibile. Milano: Etas Libri, 1998.

Zangrand, A. "Uno schema di classificazione delle organizzazioni non-profits. En Borzaga C., G. Frorentini y A. Matackna. Nonprofit e sistemi di welfare. Roma: NIS, 1996. 